\title{
Task-Based Data-Acquisition Optimization for Sparse Image Reconstruction Systems
}

\author{
Yujia Chen $^{\mathrm{a}}$, Yang Lou ${ }^{\mathrm{a}}$, Matthew A. Kupinski ${ }^{\mathrm{b}}$, and Mark A. Anastasio ${ }^{\mathrm{a}}$ \\ ${ }^{a}$ Department of Biomedical Engineering, Washington University in St. Louis, St. Louis, MO \\ 63130, USA \\ ${ }^{\mathrm{b} C o l l e g e ~ o f ~ O p t i c a l ~ S c i e n c e s, ~ T h e ~ U n i v e r s i t y ~ o f ~ A r i z o n a, ~ T u c s o n, ~ A Z ~ 85721, ~ U S A ~}$
}

\begin{abstract}
Conventional wisdom dictates that imaging hardware should be optimized by use of an ideal observer (IO) that exploits full statistical knowledge of the class of objects to be imaged, without consideration of the reconstruction method to be employed. However, accurate and tractable models of the complete object statistics are often difficult to determine in practice. Moreover, in imaging systems that employ compressive sensing concepts, imaging hardware and (sparse) image reconstruction are innately coupled technologies. We have previously proposed a sparsity-driven ideal observer (SDIO) that can be employed to optimize hardware by use of a stochastic object model that describes object sparsity. The SDIO and sparse reconstruction method can therefore be "matched" in the sense that they both utilize the same statistical information regarding the class of objects to be imaged. To efficiently compute SDIO performance, the posterior distribution is estimated by use of computational tools developed recently for variational Bayesian inference. Subsequently, the SDIO test statistic can be computed semi-analytically. The advantages of employing the SDIO instead of a Hotelling observer are systematically demonstrated in case studies in which magnetic resonance imaging (MRI) data acquisition schemes are optimized for signal detection tasks.
\end{abstract}

Keywords: Numerial observers, imaging system optimization

\section{INTRODUCTION}

It is widely accepted that optimization of imaging system performance should be guided by task-based measures of image quality, ${ }^{1}$ among which the detection of signals of interest (such as tumor or abnormal biomarkers) from background tissues is a common task of interest for doctors. For such a binary detection task, the performance of an imaging system is upper bounded by the Bayesian ideal observer (IO), ${ }^{1}$ a numerical observer employing complete knowledge of the object and noise statistics. It is widely advocated to use IO performance as a figureof-merit (FOM) to guide imaging system optimization.

However, assessment of IO performance is generally difficult. Except for rare cases, the IO test statistics do not have an explicit form and are extremely computationally intensive to evaluate. The most fundamental impediment for analytically calculating IO test statistics is the requirement to know the full probability distribution function (PDF) of the object, which often cannot be explicitly expressed in a simple way. In addition, even if a simplified object model is assumed (such as a lumpy object model ${ }^{2}$ ), the high dimensional integration in IO computation is either analytically intractable or computationally burdensome to evaluate. Sampling methods like Markov-chain Monte Carlo (MCMC) techniques have been employed to calculate the test statistics of IO. ${ }^{2}$ Although MCMC avoids the analytical calculation of the PDF and high dimensional integration, it requires considerable expertise to properly design the Monte Carlo chain, and the computation time is still prohibitively high. Therefore, although IO provides an upper bound for assessing imaging systems in performing a specific classification task, due to practical reasons, its application has been limited to relatively simple object models, and the procedure is operationally cumbersome.

Further author information: (Send correspondence to Mark A. Anastasio)

Mark A. Anastasio: E-mail: anastasio@seas.wustl.edu, Telephone: 13149353637

\footnotetext{
Medical Imaging 2017: Image Perception, Observer Performance, and Technology Assessment, edited by Matthew A. Kupinski, Robert M. Nishikawa, Proc. of SPIE Vol. 10136, $101360 Z$

(C) 2017 SPIE · CCC code: 1605-7422/17/\$18 - doi: 10.1117/12.2255536

Proc. of SPIE Vol. 10136 101360Z-1
} 
On the other hand, motivated by compressive sensing theories, modern reconstruction methods that exploit the sparsity information of the object have received wide attention in medical imaging and have proven highly effective at reconstructing images from under-sampled measurement data. The advantages of sparse reconstruction algorithms in turn enable reductions in the scanning time or radiation dose of the imaging system. In this context, a natural question for imaging system design is how to distribute resources (e.g., scanning time or radiation dose) so that the system performance for a specific task can be optimized when sparse reconstruction algorithms are employed. Since the optimal data acquisition design and the sparse reconstruction methods are fundamentally correlated, the object sparsity assumed by the reconstruction operator should also be exploited by the observer that assesses the imaging system performance.

In our previous work, we proposed a computationally tractable numerical observer, referred to as the sparsitydriven ideal observer (SDIO), ${ }^{3}$ that assumed the low-level statistical properties of the object reflecting sparsity. The SDIO and sparse reconstruction method are "matched" in the sense that they both utilizes consistent statistical information regarding the object. This match precludes the need to base ideal observer performance on object models that may not accurately represent the true object statistics. To efficiently compute SDIO performance, we estimated the posterior distribution by use of computational tools developed recently for variational Bayesian inference. ${ }^{4}$ Subsequently, the SDIO test statistic was computed semi-analytically.

The purpose of this work is to systematically investigate SDIO for guiding hardware optimization. System design by use of SDIO will be demonstrated in case studies in which MRI data acquisition schemes are optimized for signal-known-exactly/background-known statistically (SKE/BKS) signal detection tasks.

\section{CALCULATION OF SDIO TEST STATISTICS}

Consider a canonical discrete-to-discrete (D-D) imaging model described by $\boldsymbol{g}=\boldsymbol{H} \boldsymbol{f}+\boldsymbol{n}$, where $\boldsymbol{g} \in \mathbb{R}^{m}$ denotes the measurement data, $\boldsymbol{f} \in \mathbb{R}^{n}$ denotes the object to be imaged, $\boldsymbol{H} \in \mathbb{R}^{n} \rightarrow \mathbb{R}^{m}$ denotes the imaging operator, and $\boldsymbol{n} \in \mathbb{R}^{m}$ denotes random measurement noise.

We consider a SKE/BKS binary signal detection task, namely, a deterministic signal $\boldsymbol{f}_{s}$ is embedded in a random background $\boldsymbol{f}_{b}$. The binary detection task corresponds to deciding between two hypotheses: $\mathcal{H} 0: \boldsymbol{g}=$ $\boldsymbol{H} \boldsymbol{f}_{b}+\boldsymbol{n}$, and $\mathcal{H} 1: \boldsymbol{g}=\boldsymbol{H}\left(\boldsymbol{f}_{b}+\boldsymbol{f}_{s}\right)+\boldsymbol{n}$. The ideal observer calculates the likelihood ratio as its test statistic,

$$
\Lambda(\boldsymbol{g})=\frac{P(\boldsymbol{f} \mid \boldsymbol{g}, \mathcal{H} 1)}{P(\boldsymbol{f} \mid \boldsymbol{g}, \mathcal{H} 0)}
$$

where $P(\boldsymbol{f} \mid \boldsymbol{g}, \mathcal{H} i)$ is the posterior distribution under the hypothesis $\mathcal{H} i,(i=\{0,1\})$. For the SKE/BKS task, the likelihood ratio can be expressed $a^{1}$

$$
\Lambda(\boldsymbol{g})=\int d \boldsymbol{f}_{b} \Lambda_{B K E}\left(\boldsymbol{g} \mid \boldsymbol{f}_{b}\right) P\left(\boldsymbol{f}_{b} \mid \boldsymbol{g}, \mathcal{H} 0\right)
$$

where $\Lambda_{B K E}\left(\boldsymbol{g} \mid \boldsymbol{f}_{b}\right)$ is the likelihood ratio for the signal-known-exactly/background-known-exactly (SKE/BKE) case, which can often be analytically determined from the noise model. ${ }^{1}$ However, the posterior distribution $P\left(\boldsymbol{f}_{b} \mid \boldsymbol{g}, \mathcal{H} 0\right)$ is typically very difficult to compute, and the high dimensionality of the integral in Eq. (2) poses additional challenges.

The SDIO estimates the posterior distribution $P\left(\boldsymbol{f}_{b} \mid \boldsymbol{g}, \mathcal{H} 0\right)$ by adopting a variational Bayesian inference approach developed by Seeger for sparse linear models. ${ }^{4-6}$ In this study, without loss of generality, the object is assumed to be sparse in the wavelet domain. We consider a simple situation where all wavelet coefficients follow an independent and identically distributed (i.i.d.) Laplacian distribution. The prior can be expressed as

$$
P\left(\boldsymbol{f}_{b}\right)=\prod_{i=0}^{n-1} \frac{\tau_{0}}{2} \exp \left(-\tau_{0}\left|\left[\mathbf{\Phi} \boldsymbol{f}_{b}\right]_{i}\right|\right),
$$

where $\tau_{0}>0$ is a pre-determined parameter for the i.i.d Laplacian distribution reflecting the degree of sparsity, $\boldsymbol{\Phi}$ denotes the wavelet transform, and $\left[\boldsymbol{\Phi} \boldsymbol{f}_{b}\right]_{i}$ is the $i$-th element in the vector $\left[\boldsymbol{\Phi} \boldsymbol{f}_{b}\right]$. In practice, the Laplacian 
parameter $\tau_{0}$ is unknown and should be determined from a set of training data. Assume a white Gaussian noise model with a variance of $\sigma^{2}: \boldsymbol{n} \sim \mathcal{N}\left(0, \boldsymbol{\Sigma}_{n}\right)$, where $\boldsymbol{\Sigma}_{n}=\sigma^{2} \boldsymbol{I}$. According to Bayes' theorem, the posterior distribution can be written as

$$
P\left(\boldsymbol{f}_{b} \mid \boldsymbol{g}, \mathcal{H}_{0}\right)=\frac{P\left(\boldsymbol{g} \mid \boldsymbol{f}_{b}, \mathcal{H}_{0}\right) P\left(\boldsymbol{f}_{b}\right)}{\int P\left(\boldsymbol{g} \mid \boldsymbol{f}_{b}, \mathcal{H}_{0}\right) P\left(\boldsymbol{f}_{b}\right) d \boldsymbol{f}_{b}}
$$

where $P\left(\boldsymbol{g} \mid \boldsymbol{f}_{b}, \mathcal{H} 0\right)$ is the likelihood of the measurement data $\boldsymbol{g}$ given a specific object signal $\boldsymbol{f}_{b}$ with the signal absent. Given the Gaussian noise model, the likelihood function has the form of Gaussian distribution. Nevertheless, evaluating Eq. (4) with Laplacian priors still remains challenging. The variational Bayesian inference method fits the exact posterior distribution in Eq. (4) with a parameterized Gaussian function $Q\left(\boldsymbol{f}_{b}, \boldsymbol{\Gamma}\right) \sim \mathcal{N}(\boldsymbol{\mu}, \boldsymbol{\Sigma})$, where $\boldsymbol{\Gamma}$ is a diagonal matrix with parameters $\left\{\gamma_{i}\right\}(i=1, \ldots, n)$ being its diagonal elements, $\mathcal{N}$ denotes the Gaussian distribution, $\boldsymbol{\mu}=\boldsymbol{\Sigma} \boldsymbol{H}^{\dagger} \boldsymbol{\Sigma}_{n}^{-1} \boldsymbol{g}$ is the mean vector of the parameterized Gaussian function, and $\boldsymbol{\Sigma}=\left[\boldsymbol{H}^{\dagger} \boldsymbol{\Sigma}_{n}^{-1} \boldsymbol{H}+\boldsymbol{\Phi}^{\dagger} \boldsymbol{\Gamma}^{-1} \boldsymbol{\Phi}\right]^{-1}$ is the covariance matrix. The parameters $\left\{\gamma_{i}\right\}$ are chosen using a double-loop algorithm to minimize the Kullback-Leibler (K-L) divergence between Eqs. (4) and $Q\left(\boldsymbol{f}_{b}, \boldsymbol{\Gamma}\right) .{ }^{4}$

Finally, a closed-form approximation for the SDIO test statistics $\Lambda(\boldsymbol{g})$ can be expressed $\operatorname{as}^{3}$

$$
\Lambda(\boldsymbol{g})=\exp \left\{\left(\boldsymbol{g}-\frac{1}{2} \boldsymbol{H} \boldsymbol{f}_{s}\right)^{\dagger}\left(\boldsymbol{I}-\boldsymbol{\Sigma}_{n}^{-1} \boldsymbol{H} \boldsymbol{\Sigma} \boldsymbol{H}^{\dagger}\right) \boldsymbol{\Sigma}_{n}^{-1} \boldsymbol{H} \boldsymbol{f}_{s}\right\} .
$$

\section{SYSTEM RANKING CASE STUDIES}

\subsection{Simulated objects}

We applied the SDIO to rank-order several MRI data-acquisition designs based on signal detection performance. The Gaussian noise model we assumed in the last section is applicable for MRI systems. Twenty numerical 3D brain phantoms were obtained from an online phantom library, ${ }^{7}$ and from each 3D phantom, twenty $2 \mathrm{D}$ slices of $256 \times 256$ pixels were extracted, forming an ensemble of 400 background object realizations. A number of 100 randomly selected slices were employed as training samples to estimate the background covariance matrix for HO and the Laplacian parameter $\tau_{0}$ for SDIO. Another randomly selected 100 images were chosen as the test samples to test the separability of the two observers. Among the 100 test samples, 50 of the test samples corresponded to the signal present case $(\mathcal{H} 1)$ and 50 corresponded to the signal absent case $(\mathcal{H} 0)$. A deterministic artificial tumor signal was embedded in the 50 test background realizations to form $\mathcal{H} 1$ images.

\subsection{Study design}

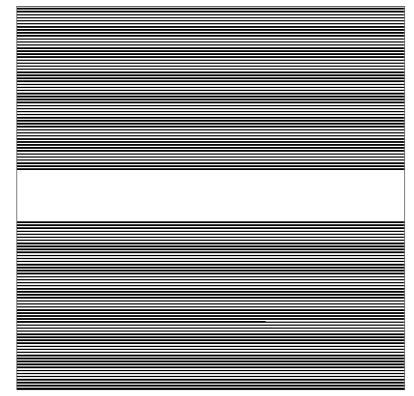

(a)

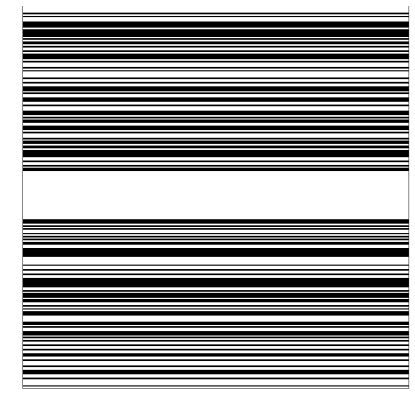

(b)

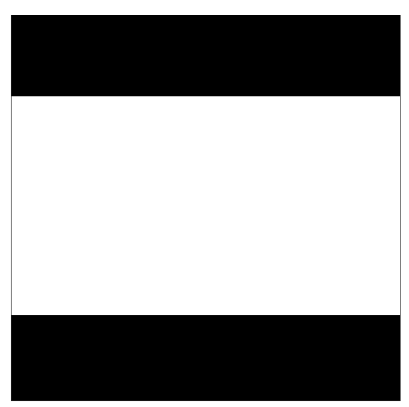

(c)

Figure 1: K-space sampling patterns : (a) Design 1: uniform half-scan; (b) Design 2: random half-scan; (c) Design 3: low-pass half-scan. The white regions are the sampled in k-space while the black regions are un-sampled. The center of the image corresponds to low-frequency components.

The goal of this simulation study was to produce a rank-ordering of data-acquisition designs for an MRI system based on signal detection performance. Three data-acquisition designs were considered. Each design containrf different phase-encoding lines in k-space, with the sampling patterns shown in Figure 1. Design 1, denoted as 
the uniform half-scan, contains 72 low-frequency lines along with 72 other uniformly sampled lines; Design 2, denoted as the random half-scan, contains 72 low-frequency lines along with 72 other randomly sampled lines with uniform distribution. Design 3, denoted as the low-pass half-scan, contains 144 low-frequency lines. For a fair comparison, all three designs contain the same number of sampled lines. Note that for all three designs, the data acquisition times are the same. The comparison can help researchers choose a better sampling design when the data acquisition time is limited.

K-space measurement data corresponding to each object were computed by applying Fourier transform and subsampling as described above. White Gaussian noise was added to the measurement data, with a standard deviation of $\sigma=2.0 \times 10^{-5}$ ( $20 \%$ of the maximum of the tumor signal intensity).

The SDIO and Hotelling observer (HO) were applied to the sets of measurement data. For the HO, the required background covariance matrix was calculated utilizing all the 100 samples in the training dataset, and its inversion was obtained by applying the matrix inversion lemma. Readers can refer to our previous work for implementation details. ${ }^{3}$ For the SDIO, only one randomly selected training sample was employed for estimating the parameter $\tau_{0}$ in fitting the Laplacian prior distribution. ${ }^{3}$ Then, the posterior distribution $P\left(\boldsymbol{f}_{b} \mid \boldsymbol{g}, \mathcal{H} 0\right)$ was fitted by the parameterized Gaussian distribution where all the parameter $\left\{\gamma_{i}\right\}$ were chosen by double-loop algorithm. ${ }^{4}$ Finally, the test statistic was computed based on Eq.(5).

The receiver operating characteristic (ROC) curves for all observers were computed based on the test dataset. For each ROC curve, the area under the curve (AUC) was calculated ${ }^{8}$ as a figure of merit to quantify the performance of the systems. All three designs were ranked based on their AUC values produced by the SDIO and $\mathrm{HO}$.

\subsection{Ranking results}

The ROC curves produced by both numerical observers are plotted in Fig. 2, with the SDIO results shown in Fig. 2a, and the HO results shown in Fig. 2b,

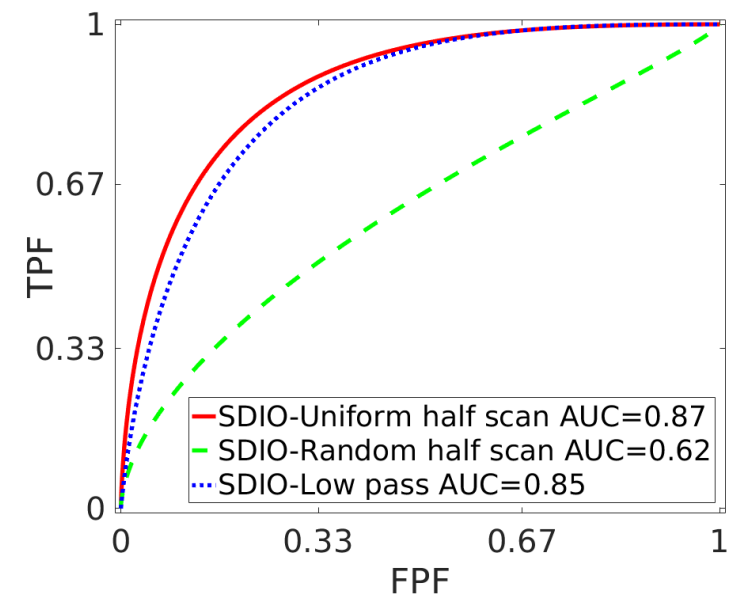

(a)

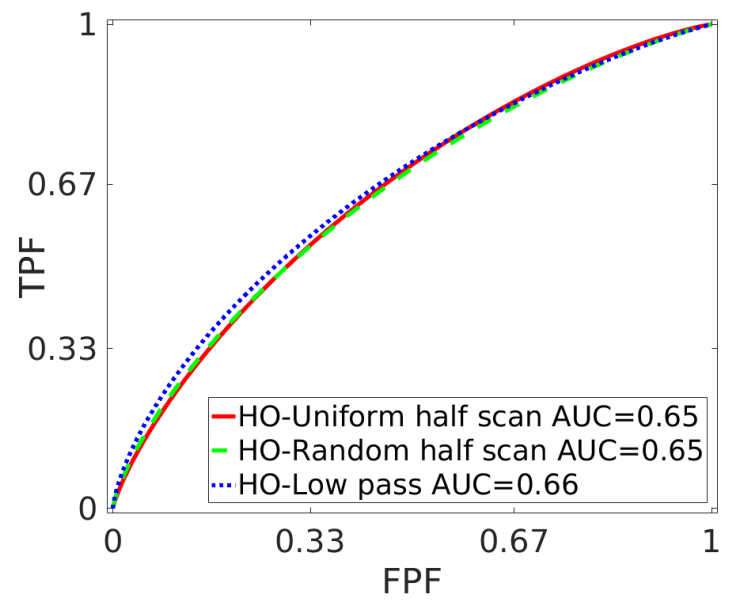

(b)

Figure 2: ROC curves with AUC for different systems from (a) SDIO, and (b) HO. Red, uniform half-scan; green, random half-scan; blue, low-pass half-scan.

The clear separability of SDIO ROC curves in Fig. 2a demonstrates that the SDIO can better differentiate the proposed candidate designs and provide a clear ranking according to their AUC values: uniform half-scan $>$ low-pass half-scan > random half-scan. For the HO, the ROC curves for different systems are quite close to each other, indicating poor separability. HO ranks low-pass half-scan $>$ random half-scan $\approx$ uniform half-scan. Among the three half-scan design options with sparse sampling, the rank-orderings produced by use of the SDIO and HO differ. Namely, the SDIO ranks the uniform half-scan as the best candidate, while the HO ranks low-pass half-scan as the best. 


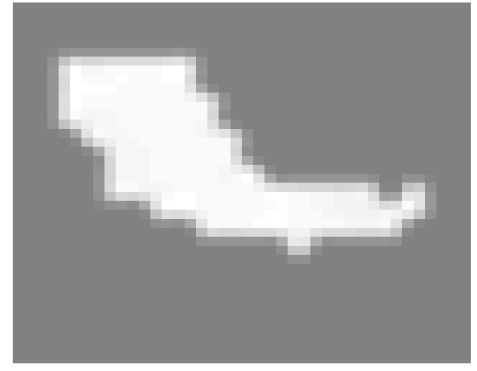

(a)

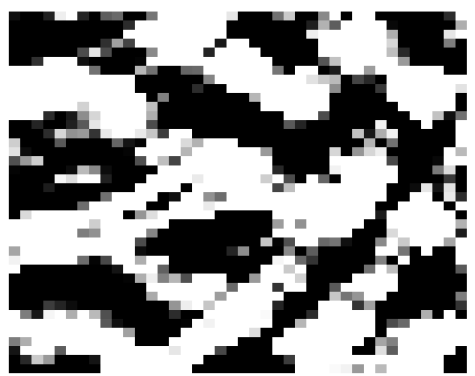

(c)

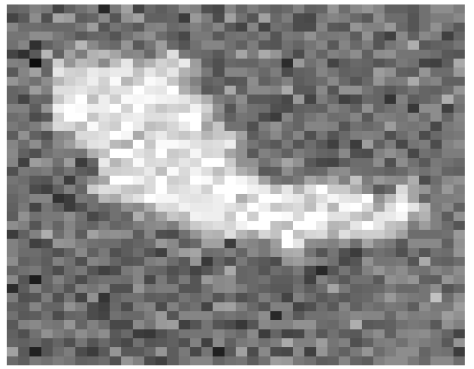

(b)

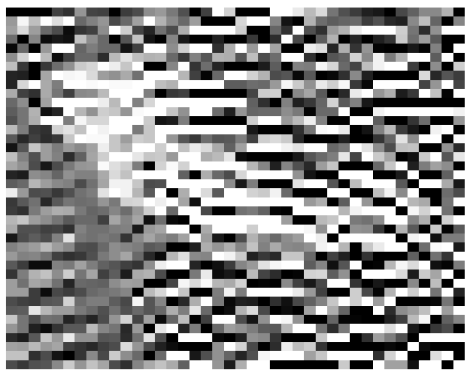

(d)

Figure 3: (a) Artificial tumor signal. (b-d) Artificial tumor signal regions of the background-removed signalpresent $(\mathcal{H} 1)$ reconstructed images for different data acquisition designs: (b) uniform half-scan; (c) random half-scan; and (d) low-pass half-scan.

Images for the signal-present case were reconstructed from data corresponding to each of the data-acquisition designs. The reconstruction algorithm computed a maximum a posteriori (MAP) estimate, where the penalty was defined as the negative logarithm of the object prior given in Eq. (3). In the reconstructed images, the signal was too weak to be reliably detected by a human observer. Therefore, for display purposes, the background was subtracted from the reconstructed images, and the corresponding regions that contained the signal are displayed in Fig. 3. These reconstruction results reveal that the signal in the uniform half-scan (subfigure (b)) case has relatively high visual contrast and is accurately recovered. The signal for low-pass half-scan (subfigure (d)) is partially obscured by artifacts and can be expected to be more difficult to detect. The signal for random half-scan (subfigure (c)) is completely obscured by artifacts. Among all three candidate systems, the signal is the most difficult to detect when random half-scan is employed, which exactly matches the SDIO ranking.

These images reconstructed by sparse reconstruction algorithm demonstrated that the sparse sampling design identified as optimal by the SDIO produced an image (Fig. 3b) in which the signal was faithfully reconstructed. On the other hand, the sparse sampling design identified as optimal by the HO produced an image (Fig. 3d) in which the signal was contaminated by artifacts. These results corroborate our claims regarding the potential advantages of the SDIO over conventional approaches for optimizing imaging systems whose measurement data will be later processed by sparse reconstruction methods. Specifically, they demonstrate that when optimizing hardware, it is desirable to utilize the same statistical information regarding the object that is implicitly assumed by the sparse image reconstruction method.

\section{CONCLUSIONS}

A newly-proposed numerical observer, referred to as the sparsity-driven ideal observer (SDIO), was investigated for guiding hardware optimization in imaging systems that employ sparse image reconstruction methods. The effectiveness of the SDIO was demonstrated in case studies in which MRI data acquisition schemes were optimized 
based on a signal detection task. The rank-ordering of data-acquisition designs based on AUC values produced by the SDIO was consistent with the visual quality of the reconstructed images, which was not true when the conventional Hotelling Observer was applied. These results demonstrate that, when optimizing systems that will employ sparse reconstruction methods, it is desirable to utilize the same statistical information regarding the object that is employed by the reconstruction method.

\section{ACKNOWLEDGMENTS}

This work was supported in part by NIH awards EB02016802 and EB02060401.

\section{REFERENCES}

[1] Barrett, H. H. and Myers, K. J., [Foundations of Image Science], John Wiley \& Sons (2013).

[2] Kupinski, M. A., Hoppin, J. W., Clarkson, E., and Barrett, H. H., "Ideal-observer computation in medical imaging with use of Markov-chain Monte Carlo techniques," JOSA A 20(3), 430-438 (2003).

[3] Wang, K., Lou, Y., Kupinski, M. A., and Anastasio, M. A., "Sparsity-driven ideal observer for computed medical imaging systems," Proc. SPIE 9416, 94161C-94161C-6 (2015).

[4] Seeger, M., Nickisch, H., Pohmann, R., and Schölkopf, B., "Optimization of k-space trajectories for compressed sensing by Bayesian experimental design," Magnetic Resonance in Medicine 63(1), 116-126 (2010).

[5] Seeger, M., "Speeding up magnetic resonance image acquisition by Bayesian multi-slice adaptive compressed sensing," Advances in Neural Information Processing Systems , 1633-1641 (2009).

[6] Seeger, M., "Gaussian covariance and scalable variational inference," Proceedings of the 27th International Conference on Machine Learning (EPFL-CONF-161304), Omnipress (2010).

[7] Elad, M., [Sparse and Redundant Representations: From Theory to Applications in Signal and Image Processing], Springer (2010).

[8] Metz, C. E. and Kronman, H. B., "Statistical significance tests for binormal ROC curves," Journal of Mathematical Psychology 22(3), 218-243 (1980). 